\title{
Disrupted Frontoparietal Network Mediates White Matter Structure Dysfunction Associated with Cognitive Decline in Hypertension Patients
}

\author{
Xin Li, ${ }^{1,2 *}$ Ying Liang, ${ }^{1,2 *}$ Yaojing Chen, ${ }^{1,2 *}$ Junying Zhang, ${ }^{1,2}$ Dongfeng Wei, ${ }^{1,2,3}$ Kewei Chen, ${ }^{1,4}$ Ni Shu, ${ }^{1,2}$ \\ Eric M. Reiman, ${ }^{4}$ and Zhanjun Zhang ${ }^{1,2,3}$ \\ ${ }^{1}$ State Key Laboratory of Cognitive Neuroscience and Learning and IDG/McGovern Institute for Brain Research, ${ }^{2}$ Beijing Aging Brain Rejuvenation \\ Initiative Centre, Beijing Normal University, Beijing 100875, China, ${ }^{3}$ Institute of Basic Research in Clinical Medicine, China Academy of Traditional Chinese \\ Medicine, Beijing 100700, China, and 4Banner Alzheimer's Institute, Phoenix, Arizona 85006
}

\begin{abstract}
Some previous reports have suggested that hypertension is a risk factor for dementia and cognitive impairments. Using behavioral data from 1007 elderly human subjects ( 405 hypertensive patients) of Han ethnicity from Beijing, China, the present study aimed to assess the effects of hypertension on cognitive performance and explore related neuronal changes via advanced resting-state functional magnetic resonance imaging and diffusion tensor imaging data from 84 of these subjects ( 44 hypertensive patients). Cognitively, we found that patients with hypertension showed decreased executive functions and attention compared with those with normotension in the large sample. In magnetic resonance imaging scan sample, using independent component analysis to examine the functional connectivity difference between the two groups, we found that the frontoparietal networks in the hypertensive group exhibited altered patterns compared with the control group, mainly in the inferior parietal lobe, left inferior frontal lobe, and precuneus. Using tract-based spatial statistics to investigate the between-group structural difference, we found that the hypertensive group showed significantly reduced integrity of white matter in the bilateral superior longitudinal fasciculus. Importantly, using the mediation analysis, we found that the functional connectivity of the frontoparietal networks mediates the impact of white matter on executive function in the hypertensive group. The results demonstrate that hypertension targets a specific pattern of cognitive decline, possibly due to deficits in the white matter and functional connectivity in frontal and parietal lobes. Our findings highlight the importance of brain protection in hypertension.
\end{abstract}

Key words: hypertension; frontoparietal networks; functional connectivity; superior longitudinal fasciculus

Significance Statement

Hypertension is a risk factor for cognitive decline and dementia. However, the neural mechanism underlying cognitive decline in hypertension is largely unknown. We studied the relationship among cognitive decline, brain functional, and structural changes in hypertensive patients via advanced resting-state functional magnetic resonance imaging and diffusion tensor imaging data in a Chinese cohort. Hypertensive patients showed executive dysfunction, along with disrupted functional connectivity in frontoparietal (FP) networks and reduced integrity of white matter in the bilateral superior longitudinal fasciculus. Importantly, the functional connectivity changes mediate the impact of white matter alterations on cognitive decline in the hypertensive group. Our findings provide a better understanding of the mechanism of cognitive decline in hypertension and highlight the importance of brain protection in hypertension.

\section{Introduction}

In China, hypertension is a disease of pandemic proportions. In addition to leading to serious cardiovascular consequences, such as stroke, myocardium hypertrophy, and heart failure, hyperten-

Received Dec. 16, 2014; revised June 3, 2015; accepted June 5, 2015.

Author contributions: X.L., Y.L., K.C., and Z.Z. designed research; J.Z. and D.W. performed research; Z.Z. contributed unpublished reagents/analytic tools; X.L., Y.L., Y.C., N.S., and Z.Z. analyzed data;X.L.,Y.C., K.C., N.S., E.M.R., and Z.Z. wrote the paper. sion is also considered to be a pivotal risk factor for dementia (Wiseman et al., 2004; Sepe-Monti et al., 2007). However, there is limited evidence regarding the neural mechanism of cognitive decline in hypertension. 
The panoply of cognitive functions that underpin everyday human experience requires precisely choreographed patterns of interaction among networked brain regions (Mesulam, 1998; Leech et al., 2011). Previous research has reported that there are some functional resting-state networks (RSNs) in the brain (Sorg et al., 2007). As the most important and common RSNs, default mode networks (DMNs) mainly underpin the core social domains of cognition, such as self-representation and mentalization (Tsioufis et al., 2006; Buckner et al., 2008); the FP network mainly underpins the core executive domains of cognition (Assmus et al., 2007; Bartolomeo et al., 2007; Leech et al., 2011). Aberrant connectivity patterns are evident across brain disorders (Leech et al., 2011). In Alzheimer's disease (AD; Sperling et al., 2010) and mild cognitive impairment (Weiler et al., 2014), there is a changed DMN that is related to impaired memory ability. These RSNs may play a role in cognitive function impairments in related diseases. However, no study has investigated changes in RSNs in hypertensive patients.

Functional connectivity (FC) is based on the white matter tract connectivity to facilitate ongoing inter-regional neuronal communication (van den Heuvel et al., 2009). The cingulum tract plays an important role in the FC of DMNs (Park et al., 2005; Schlosser et al., 2005). In patients with multiple sclerosis, Lowe et al. demonstrated that decreases of FC between the left and right primary motor regions are associated with the changed corpus callosum tracts (Le et al., 2005). Furthermore, van den Heuvel et al. (2009) have reported that regions within RSNs are densely interconnected by white matter fiber tracts. Together, these results suggest that the effect of widespread white matter tract deficits is likely to be a key factor that affects brain functional network connectivity.

Thus, studying the domains of cognitive impairment due to hypertension, one of the most important risk factors for dementia, could help in understanding the hypertension-related neural mechanism and in finding early warning signs for dementia and ways to delay the development of pathophysiological deficits in the brain. We first assessed the cognitive performance of hypertensive patients with a battery of neuropsychological tests in a large sample of people of Han ethnicity and the related neural mechanism. For the hypertension-related pathophysiologic brain alterations, we then chose a representative sample for magnetic resonance imaging (MRI) scanning and combined restingstate functional MRI (fMRI) and diffusion tensor imaging (DTI) technology to investigate the functional and structural connectivity of RSNs in hypertensive patients.

\section{Materials and Methods Large sample of behavioral research Participants}

The participants in the present study were from the Beijing Aging Brain Rejuvenation Initiative (BABRI) and were recruited for a cross-sectional study of urban elderly adults in Beijing, China (Nielsen, 1975). People with suspected dementia or who were unable to complete the neuropsychological tests because of physical or mental disability were excluded from the study. People who completed the neuropsychological tests but

and Youth Scholars Program of Beijing Normal University (Grant \#2014NT19). We thank all the volunteers and participants for their participation in our study and Lu Zheng, Lei Qiu, Xiaotang Zhu, Yao Zhang, and Tianjiao Feng for community contact and acquisition of data.

The authors declare no competing financial interests.

*X.L., Y.L., and Y.C. contributed equally to this work.

Correspondence should be addressed to Zhanjun Zhang, MD, State Key Laboratory of Cognitive Neuroscience and Learning, Beijing Normal University, Beijing 100875, China. E-mail: zhang_rzs@bnu.edu.cn.

DOI:10.1523/JNEUROSCI.5113-14.2015

Copyright $\odot 2015$ the authors $\quad 0270-6474 / 15 / 3510016-10 \$ 15.00 / 0$ refused to answer the personal information questionnaire for privacy or other reasons were excluded from the analysis of associated factors. We excluded participants with structural abnormalities that could impair cognitive function; these included cerebrovascular lesions, such as tumor, subdural hematoma, and contusion due to a previous head trauma. We also excluded participants with addictions, psychiatric diseases, or those undergoing treatments that would affect cognitive function (such as antidepressants, pain medications, sleep aids, benzodiazepines, etc.). All of the hypertensive patients were diagnosed by a physician and had a history of using oral antihypertensive medications based on their medical records. There were 1007 participants who completed the neuropsychological tests and personal information questionnaire. Among them, 405 participants (264 females) reported that they were diagnosed with hypertension and 602 participants ( 358 females) reported no history of hypertension according to their medical records (Table 1). The study was approved by the institutional review board of Beijing Normal University.

Neuropsychological tests and personal information questionnaire A group of students trained by professional neuropsychologists performed the neuropsychological tests and the personal information questionnaire. The Chinese translation of the Mini-Mental State Examination (MMSE) served as a general cognitive function test. The subsequent neuropsychological battery was tested in five cognition domains: (1) memory, tested by the Auditory Verbal Learning (AVLT-delay recall; Rosenberg et al., 1984) and the Rey-Osterrieth Complex Figure tests (ROCF-delay recall, Tupler et al., 1995); (2) visuospatial ability and attention, tested by the ROCF-copy test (Tupler et al., 1995), the clockdrawing test (Ishiai et al., 1993), and the Symbol Digit Modalities Test (SDMT; Sheridan et al., 2006); (3) language, tested by the Category Verbal Fluency Test (Mok et al., 2004) and the Boston Naming Test Knesevich et al., 1986); (4) processing speed, tested by the Stroop Test (Stroop B-time) and the Trail Making Test A (TMTa); and (5) executive function, tested by the Trail Making Test B-A (TMTba; Gordon, 1972) and the Stroop Test (Stroop CB-time; Koss et al., 1984).

The personal information questionnaire included demographic information and medical history. Demographic information included age, gender, and years of formal education. Medical history included questions on a series of chronic diseases, including hypertension, coronary heart disease, diabetes mellitus, cerebrovascular disease, chronic bronchitis or emphysema, osteoarthritis, and intervertebral disk disease.

\section{MRI studies}

\section{Participants}

We choose 44 hypertensive patients ( 21 females) from BABRI to undergo MRI scanning. All MRI images of the small sample were acquired $\leq 1$ month after the neuropsychological tests. All of the hypertensive patients were diagnosed by a physician and had a history of using oral antihypertensive medications. The diastolic and systolic blood pressures were measured with standard laboratory testing by a specialist physician. The blood pressures for all patients were controlled below 140/90 $\mathrm{mmHg}$. The duration of hypertension was defined as the number of years since diagnosis. The antihypertensive medications included angiotensin receptor blockers, calcium channel blocker, diuretics, $\beta$ blockers, and compound antihypertensives. All participants had a medical history and physical examination, during which height, weight, and body mass index were recorded. Blood samples were collected at Beifang hospital. The serum total cholesterol, triglyceride, high-density lipoprotein, lowdensity lipoprotein, and fasting plasma glucose levels were measured with an Automatic Biochemistry Analyzer (Olympus AU400) using commercially available diagnostic kits (Beckman Coulter). Exclusion criteria for the groups were a previous history of chronic disease (including coronary heart disease, diabetes mellitus, or cerebrovascular disease), neurological disease (including stroke, dementia, or transient ischemic attack), and unsuitability for MRI (e.g., due to a pacemaker, prosthetic heart valve, or claustrophobia). All participants gave written informed consent to our protocol, which was approved by the ethics committee of the State Key Laboratory of Cognitive Neuroscience and Learning, Beijing Normal University. All subjects were right handed and native Chinese speakers. 
Table 1. Characteristics of all participants and neuropsychological test results in large sample from Beijing communities

\begin{tabular}{|c|c|c|c|c|c|c|}
\hline & \multicolumn{2}{|c|}{ Hypertension $(n=405)$} & \multicolumn{2}{|c|}{ Control $(n=602)$} & \multirow[b]{2}{*}{$F / t / \chi^{2}$} & \multirow[b]{2}{*}{$p$} \\
\hline & Mean & SD & Mean & SD & & \\
\hline Age (years) & 64.87 & 6.586 & 64.46 & 6.752 & 0.34 & 0.732 \\
\hline Education (years) & 11.02 & 3.516 & 11.27 & 3.440 & -1.04 & 0.302 \\
\hline Gender (male/female) & \multicolumn{2}{|c|}{$141 / 264$} & \multicolumn{2}{|c|}{$244 / 358$} & 3.35 & 0.074 \\
\hline MMSE & 27.47 & 2.169 & 27.89 & 1.924 & 8.97 & 0.003 \\
\hline \multicolumn{7}{|l|}{ Executive function } \\
\hline Stroop CB & 41.10 & 18.893 & 36.93 & 18.871 & 6.71 & 0.010 \\
\hline TMTba & 124.83 & 59.788 & 112.45 & 56.194 & 4.07 & 0.044 \\
\hline \multicolumn{7}{|l|}{ Processing speed } \\
\hline Stroop B-time & 40.06 & 11.051 & 39.12 & 12.305 & 0.04 & 0.847 \\
\hline TMTa & 61.66 & 23.041 & 59.81 & 24.138 & 0.10 & 0.748 \\
\hline \multicolumn{7}{|l|}{ Memory } \\
\hline AVLT-delay recall & 5.44 & 2.525 & 5.87 & 2.519 & 5.11 & 0.024 \\
\hline ROCF-delay recall & 12.69 & 6.374 & 13.57 & 6.117 & 1.15 & 0.283 \\
\hline \multicolumn{7}{|l|}{ Language } \\
\hline Category Verbal Fluency Test & 44.20 & 8.427 & 44.94 & 9.027 & 0.65 & 0.421 \\
\hline Boston Naming Test & 23.10 & 3.801 & 23.31 & 3.910 & 0.02 & 0.889 \\
\hline \multicolumn{7}{|l|}{ Visual-spatial and attention } \\
\hline ROCF-copy & 32.85 & 3.890 & 33.52 & 3.490 & 3.66 & 0.056 \\
\hline Clock-drawing test & 24.47 & 4.127 & 25.10 & 3.938 & 3.53 & 0.061 \\
\hline SDMT & 33.44 & 10.638 & 35.21 & 11.339 & 3.23 & 0.073 \\
\hline
\end{tabular}

The comparisons of age and education were performed with independent two-sample $t$ test. The $p$ value for gender was obtained using a $\chi^{2}$ test. Neuropsychological assessment differences between the two groups were analyzed with an ANCOVA, with age, education, gender, and medical history as covariates.

Table 2. Characteristics and neuropsychological test results of MRI study participants

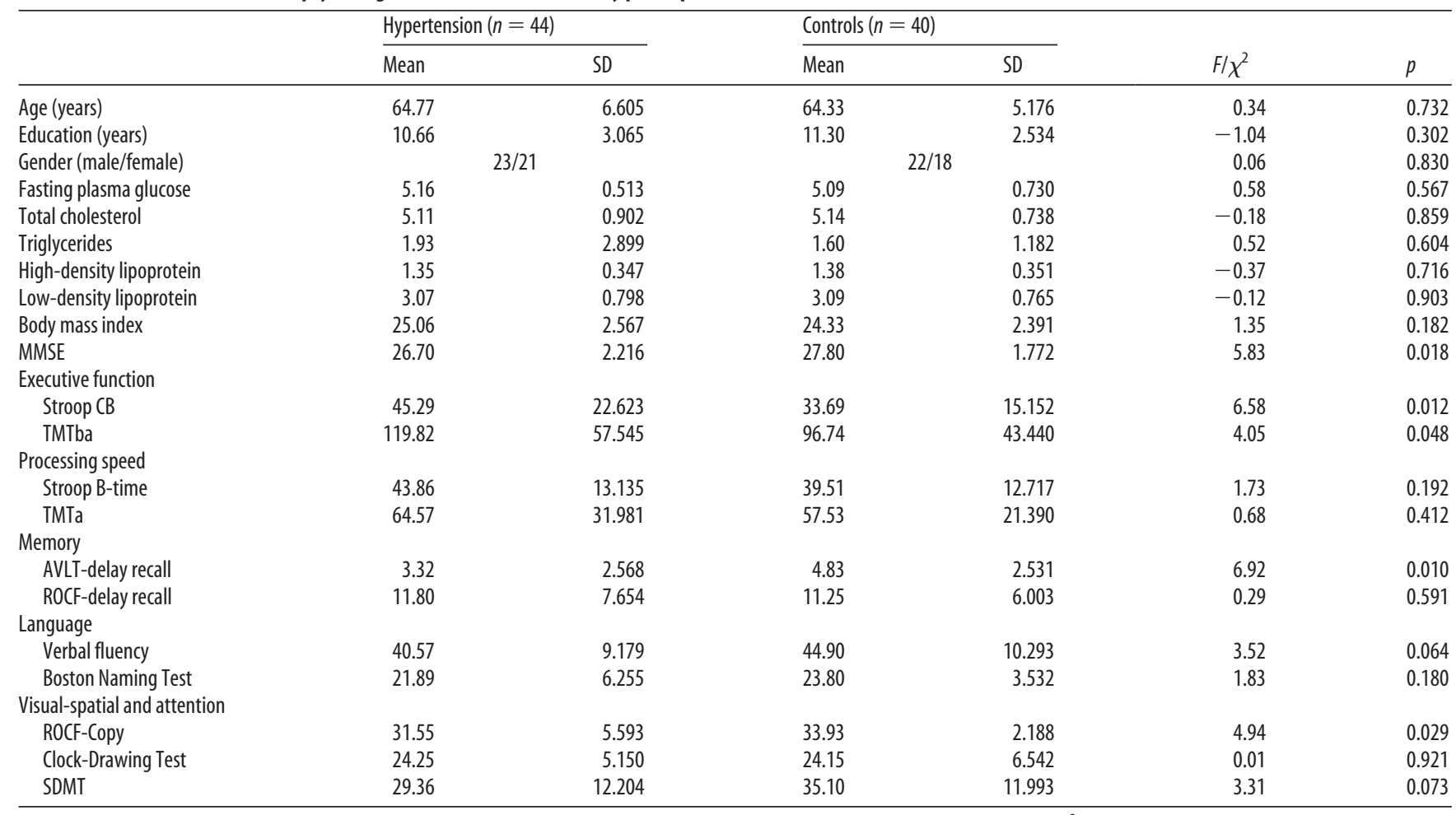

The comparisons of neuropsychological test were performed with an ANCOVA, with age, education, and gender as covariates. The $p$ value for gender was obtained using a $\chi^{2}$ test.

Forty healthy subjects (18 females) who reported no history of vascular, psychiatric, or neurological problems were included as an agematched and sex-matched control group (Table 2).

MRI data acquisition

MRI data were acquired using a Siemens Trio 3T scanner in the Imaging Center for Brain Research, Beijing Normal University, and included high-resolution T1-weighted scans, high-resolution T2-weighted scans, and fluid-attenuated inversion recovery and resting-state functional magnetic resonance imaging (rsfMRI) scans. Participants laid supine with their head snugly fixed by straps and foam pads to minimize head movement. T1-weighted, sagittal 3D magnetization-prepared rapid gradient echo sequences were acquired and covered the entire brain [176 slices; repetition time $(\mathrm{TR})=1900 \mathrm{~ms}$; echo time $(\mathrm{TE})=3.44 \mathrm{~ms}$; slice thickness, $1 \mathrm{~mm}$; flip angle, $9^{\circ}$; inversion time, $900 \mathrm{~ms}$; field of view, $256 \times 256 \mathrm{~mm}^{2}$; acquisition matrix, $\left.256 \times 256\right]$. Resting-state data were collected using a gradient echo EPI sequence $(\mathrm{TE}=30 \mathrm{~ms}$; TR $=2000$ $\mathrm{ms}$; flip angle, $90^{\circ}$; 33 slices; slice thickness, $4 \mathrm{~mm}$; matrix, $\left.64 \times 64\right)$. DTIs were acquired using a single-shot EPI sequence [coverage of the whole brain; $2 \mathrm{~mm}$ slice thickness with no interslice gap; 70 axial slices; TR $=$ $9500 \mathrm{~ms}$; TE $=92 \mathrm{~ms}$; flip angle, $90^{\circ} ; 30$ diffusion directions with $b=$ 


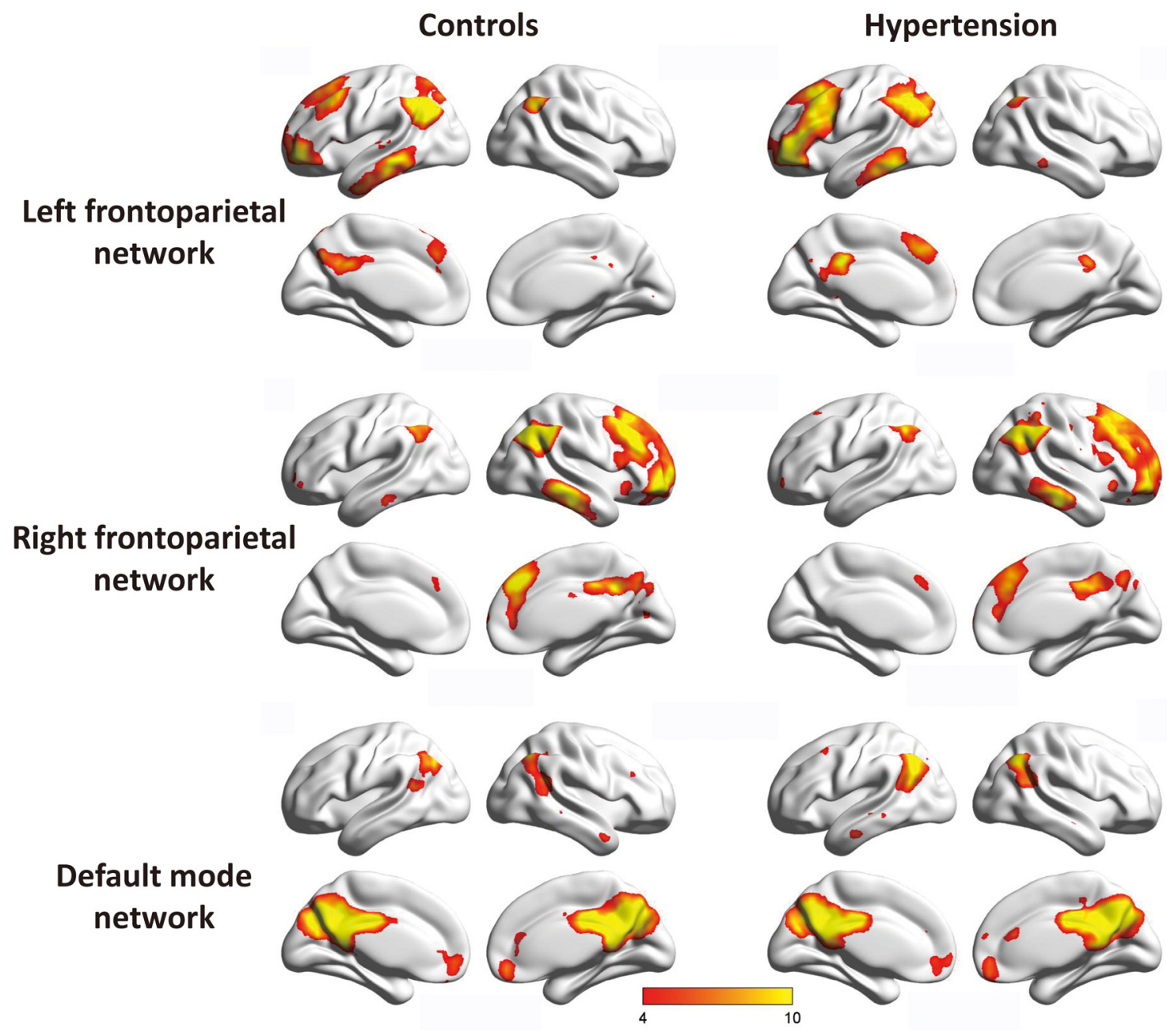

Figure 1. Group ICA estimated resting-state patterns grouped as the LFP network, RFP network, and DMN in each group (color-coded $t$ value).

$1000 \mathrm{~s} / \mathrm{mm}^{2}$ and an additional image without diffusion weighting (i.e., $b=0 \mathrm{~s} / \mathrm{mm}^{2}$ ); acquisition matrix, $128 \times 128$; field of view, $256 \times 256$ $\mathrm{mm}^{2}$; averages, 3].

Rest-fMRI data processing

Preprocessing. For each participant, the first 10 volumes were discarded to allow the participants to adapt to the magnetic field. Functional data were preprocessed using SPM8 (http://www.fil.ion.ucl.ac.uk/spm) and Data Processing Assistant for Resting-State fMRI (http://www.restfmri.net/ forum/taxonomy/term/36), including slice timing, within-subject interscan realignment to correct possible movement, spatial normalization to a standard brain template in the Montreal Neurological Institute (MNI) coordinate space, resampling to $3 \times 3 \times 3 \mathrm{~mm}^{3}$, and smoothing with an $8 \mathrm{~mm}$ full-width half-maximum Gaussian kernel. In addition, rsfMRI data were processed with linear detrending, 0.01-0.08 Hz bandpass filtering, and regression correction for nuisance covariates including six motion parameters, the global mean signal, the white matter signal, and the CSF signal.

Independent component analysis. We performed an independent component analysis (ICA) using the group ICA toolbox (GIFT version 2.0e; http://mialab.mrn.org/software/gift/) with 25 independent components separately estimated for each group. There are three main stages: (1) principal component analysis was performed for each subject for data reduction, (2) application of the ICA algorithm, and (3) back reconstruction for each individual subject (Calhoun et al., 2001). The best-fit components for the left FP (LFP) network, right FP (RFP) network, and $\mathrm{DMN}$ were identified by visual inspection. Based on the previous studies, the bilateral FP networks and DMNs are particularly relevant with cog- nitive impairments (Sorg et al., 2007; Sun et al., 2011). For each network, ANCOVA tests were used to compare FC $z$ values to determine the significance of between-group differences $[q<0.05$, false discovery rate (FDR) corrected].

\section{DTI data processing}

Imaging preprocessing. Preprocessing of the imaging data consisted of three steps. First, eddy current distortions and motion artifacts were corrected by applying affine alignment of each diffusion-weighted image to the $b=0$ image using Functional Magnetic Resonance Imaging of the Brain's (FMRIB's) Diffusion Toolbox [FDT; FMRIB Software Library (FSL) 4.1.4; www.fmrib.ox.ac.uk/fsl]. The first volume of the diffusion data without a gradient applied (i.e., the $b=0$ image) was then used to generate a binary brain mask using the Brain Extraction Tool. Finally, DTI fit was used to independently fit a diffusion tensor to each voxel. The output of DTI fit yielded voxelwise maps of fractional anisotropy (FA) and mean diffusivity (MD). Then, to allow for atlas-based analysis, DTI images of native space were registered to a standardized template in MNI using FLIRT (FLRIB's Linear Registration Tool) command of FSL.

Atlas-based quantification at the tract level. To investigate the diffusion changes in several specific tracts, we adopted the atlas-based segmentation strategy, and a widely used white matter tractography atlas, the Johns Hopkins University (JHU) White Matter Atlas (cmrm.med.jhmi.edu/), was applied. We calculated the regional diffusion metrics by averaging the values in each region of the atlas in the standard space. The JHU White Matter Atlas contains 20 major white matter fiber tracts in the brain: the anterior thalamic radiation, the corticospinal tract, the cingulum, the cingulum (hippocampus part), the forceps major, the forceps 


\section{Control>Hypertension}

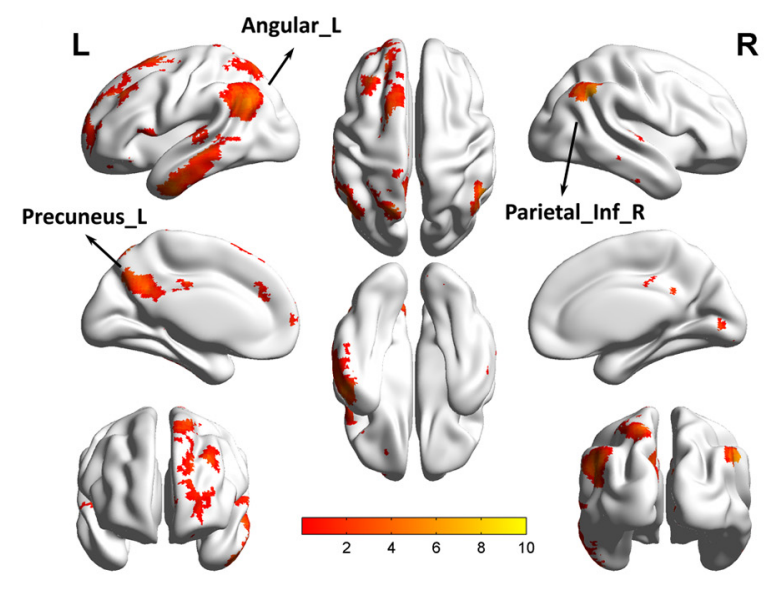

$\mathbf{R}$

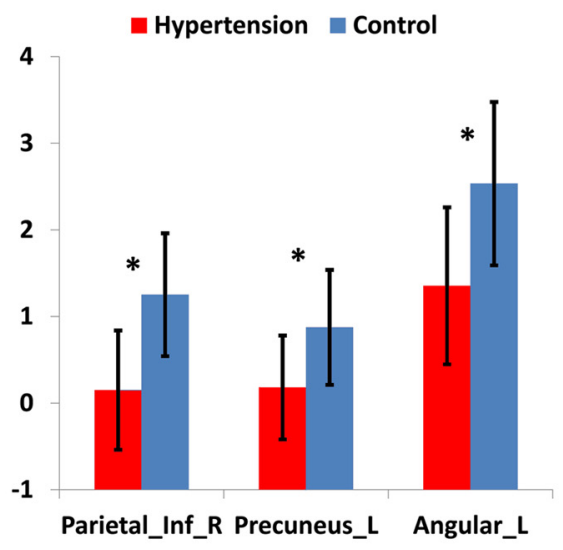

Control<Hypertension
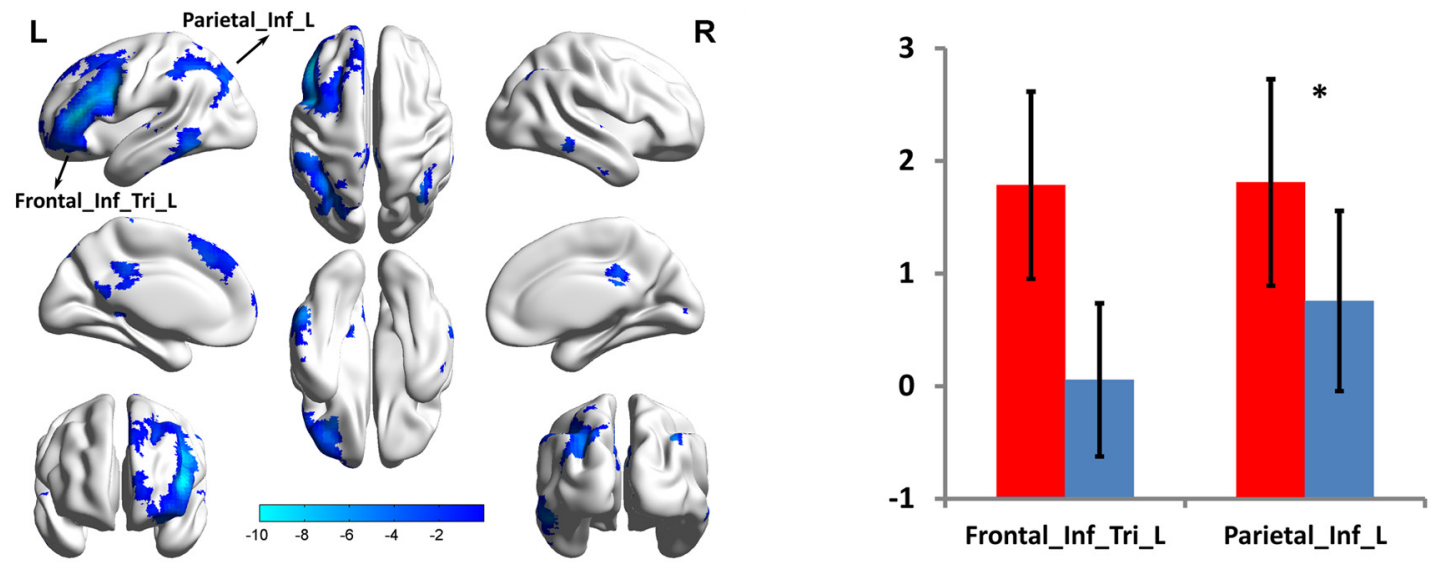

Figure 2. FC within the LFP network. Top, Voxels showing a lower correlation with the network-specific time course of the LFP network for patients with hypertension than controls (red; including the left angular, left precuneus, and right inferior parietal lobe). Bottom, Greater correlations in the LFP network in hypertension (blue; including the left inferior parietal lobe and left inferior frontal gyrus).

minor, the inferior fronto-occipital fasciculus, the inferior longitudinal fasciculus, the superior longitudinal fasciculus (SLF), the uncinate fasciculus, the uncinate fasciculus, and the temporal part of SLF. Average values of FA and MD were computed for these major tracts in each participant.

\section{Mediation analysis}

The mediation analyses were used by the Hayes Process macro (Hayes, 2013; http://www.afhayes.com/). This macro runs a series of ordinary least-squares regressions with the centered product term. In these regressions, the independent factor was white matter integrity, the dependent variable was the cognitive measure that showed significant group differences, and the proposed mediator was the FC of the five ROIs that also showed a significant group effect. These regressions also included the direct model of white matter on cognition, and the mediation terms (FC of left networks) to determine whether it played an important role in governing the association between white matter and cognition. Mediation analyses controlled for age, gender, and education. Statistical significance was set at 0.05 and SPSS versions 17.0 were used for all analyses.

\section{Statistical analysis}

Neuropsychological assessment differences between the two groups were analyzed with the ANCOVA, with age, education, gender, and medical history as covariates from sample of 1007 subjects. Partial correlation analyses were performed separately for the hypertensive and control individuals to explore the relationship between white matter integrity (or
FC of networks) and neuropsychological performance, with age, education, and gender as covariates from MRI scan sample. These associations were significant if $p$ was $<0.05$. All statistical analyses were performed using SPSS version 17.0 for Windows.

\section{Results}

Neuropsychological characteristics of the large sample In the large sample, there were no significant differences in age, years of education, and gender $(p>0.05)$. The hypertensive patients had worse performance of executive function (Stroop $\mathrm{CB}, F=6.71, p=0.010$; TMTba, $F=4.07, p=0.044)$, memory (AVLT, $F=5.11, p=0.024)$, and $\operatorname{MMSE}(F=8.97, p=0.003)$ compared with controls. Although other cognitive domains revealed no difference between groups, we found a trend for cognitive decline in the patients (Table 1 ).

\section{Selectively altered RSNs in patients with hypertension}

To investigate the pattern of the intrinsic FC of hypertension patients, the best-fit components for the LFP networks, RFP networks, and DMNs were obtained by the ICA group (Fig. 1). We performed an ANCOVA test on each of the three RSNs contrasting the individual, back-reconstructed independent component patterns of both groups. Age, gender, and education were considered as covariates. The LFP network revealed a significant group 

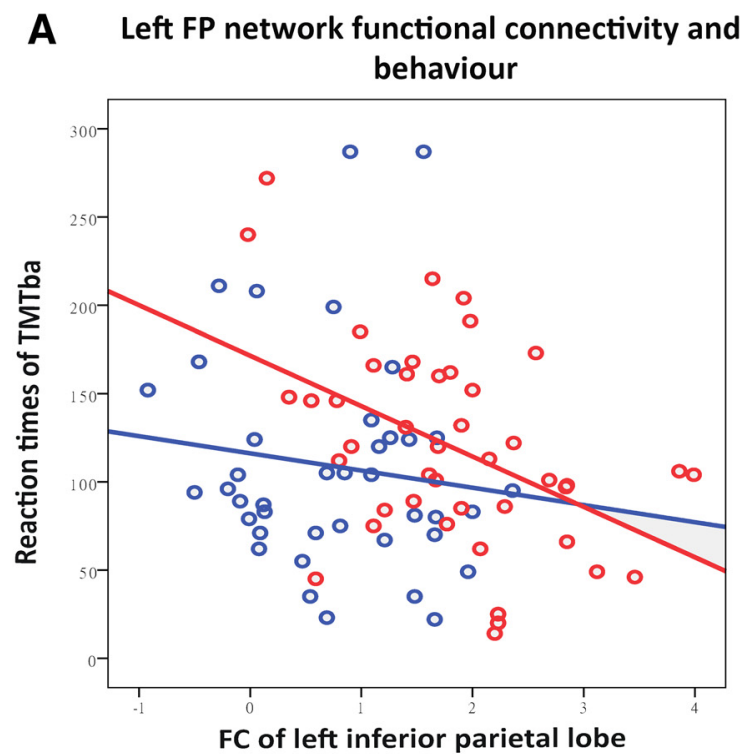

o Hypertension

\section{B Left SLF integrity and Left FP network} functional connectivity

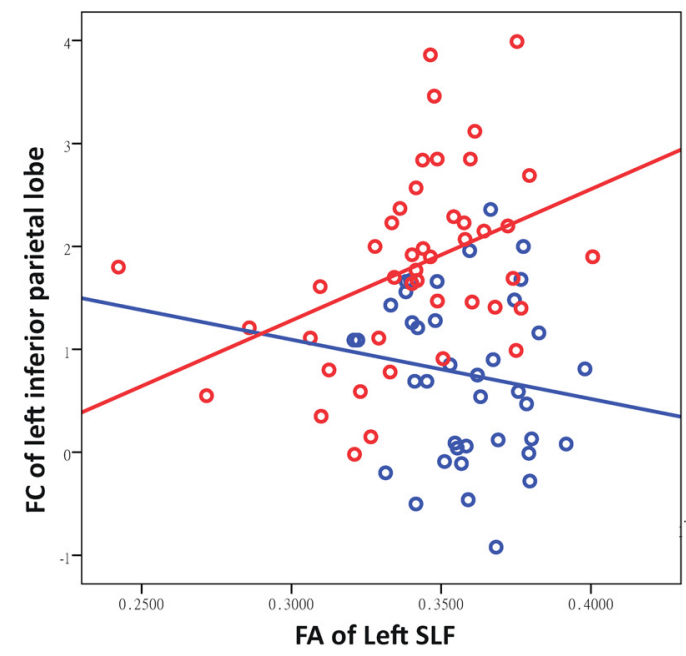

Figure 3. The relationship among white matter integrity, FC, and cognitive performance in LFP networks. $A$, Left inferior parietal lobe FC within the LFP network is correlated with the reaction times of TMTba. Left inferior parietal lobe $\mathrm{FC}$ is plotted against median reaction time on the reaction time TMTba task. $\boldsymbol{B}$, The correlation of white matter integrity in the left SLF as measured by FA and the left inferior parietal lobe $\mathrm{FC}$ with the rest of the LFP network. To avoid bias, the left inferior parietal lobe was sampled from the peak of the LFP network in the reference independent component analysis $(x=-33, y=-57, z=45)$. Patients and controls are plotted separately. Hypertension is plotted in red and controls in blue circles.

difference $[p$ (FDR-corrected for multiple comparisons) $<0.01]$. In the right inferior parietal lobe, left precuneus, and left angular areas of the LFP networks, associated independent component patterns demonstrated reduced connectivity in patients with hypertension. Meanwhile, in the left inferior parietal lobe and left triangle inferior frontal gyrus areas, increased connectivity was detected in the patient group (Fig. 2). None of the ANCOVA tests on the RFP networks and DMNs revealed a significant group difference $[p$ (FDR-corrected for multiple comparisons $)<0.01]$.

\section{FP network FC is correlated with behavior}

Greater FC within the RSNs may allow for more efficient network function (Davies et al., 2011). To investigate this possibility, we tested whether variability in the LFP network FC at "rest" predicted cognitive performance. We examined the relationship between FC of different regions and cognitive behavior. The results showed that only the FC of the left inferior parietal lobe is significantly correlated with TMTba $(r=-0.456, p=0.002$; Fig. $3 A)$, $\operatorname{SDMT}(r=0.371, p=0.013)$, and verbal fluency $(r=0.335, p=$ $0.026)$ in the hypertensive group. There was no significant correlation in the control groups.

\section{Group comparisons of atlas-based tract ROIs}

Compared with controls, hypertension patients showed significantly reduced FA across several white matter regions, including right anterior thalamic radiation $(F=6.34, p=0.014)$, left cingulum cingulated gyrus $(F=5.91, p=0.017)$, forceps major $(F=$ 6.87, $p=0.011)$, left SLF $(F=10.45, p=0.002)$, and right SLF $(F=11.09, p=0.001)$. Hypertension patients showed significantly increased MD across several white matter regions, including bilateral anterior thalamic radiation (left, $F=7.37, p=0.008$; right, $F=8.00, p=0.006$ ), bilateral corticospinal tract (left, $F=$ $6.58, p=0.012$; right, $F=5.70, p=0.019)$, forceps major $(F=$ 5.73, $p=0.019)$, forceps minor $(F=6.21, p=0.015)$, right inferior fronto-occipital fasciculus $(F=8.00, p=0.006)$, and bilateral SLF (left, $F=7.37, p=0.008$; right, $F=11.50, p=0.001$;
Fig. 4). FDR correction was used for multiple comparisons $(q<$ 0.05). Age, education, and gender were covariates.

\section{White matter structure correlates with FP network} connectivity

FA in the left SLF was positively correlated with left inferior parietal lobe FC $(r=0.68, p=0.001)$, corrected for multiple comparisons in hypertension (Fig. $3 B$ ). Therefore, patients with more disrupted white matter integrity showed less FC within the LFP network. There was no significant correlation in controls.

\section{Mediation analysis}

In the mediation analysis, the independent factor was white matter integrity, which showed significant group differences (such as the anterior thalamic radiation, cingulated gyrus, forceps major, and SLF), and the dependent variables were cognitive measures that showed significant group differences, such as Stroop CB and TMTba. The proposed mediator was the FC of the LFP network, which showed significant hypertension effects in the above $t$ test analyses. As shown in Figure 5, mediation analysis indicated that the FC of left inferior parietal in LFP networks mediates the effect of FA of left SLF variants on TMTba $(Z=-2.0136, p=0.0440$; Fig. 5).

\section{Discussion}

The study assessed the effects of hypertension on cognitive performance in a large sample from the Beijing community. Cognitively, hypertensive patients mainly showed decreased execution functions compared with the controls in the large sample. To investigate the neural mechanisms of the cognitive deficits, we studied the key cognitive brain networks, including LFP networks, RFP networks, and DMNs. In the LFP networks, there were obvious abnormal patterns of FC in hypertensive patients. The important finding was that the SLF, which interconnects the FP networks, showed changed white matter integrity. More interestingly, the mediation analysis indicated that reduced FA in left SLF may influence cognitive decline via the alteration of the 
A

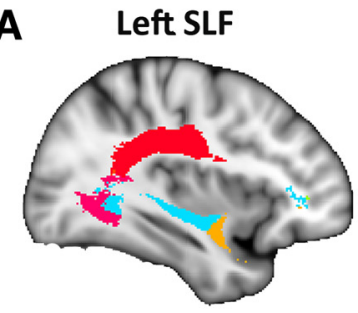

B

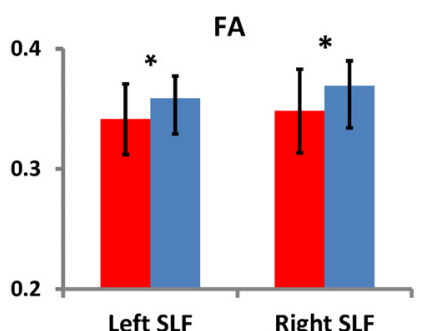

Hypertension

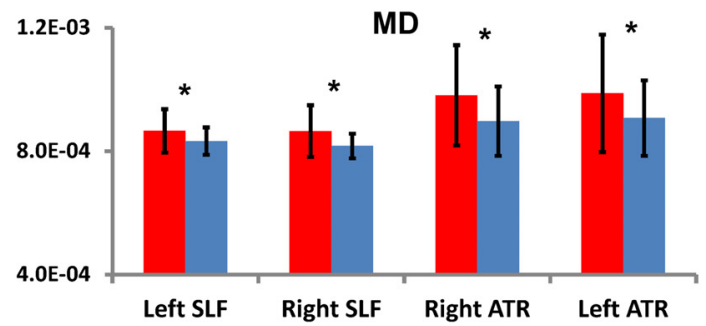

Healthy Controls

Figure 4. Mean diffusion metrics of the atlas-based tracts in hypertension and control groups. $A$, The JHU White Matter Atlas was overlaid on the mean white matter skeleton in the ICBM-152 space. Colored regions indicate major white matter tracts. $\boldsymbol{B}$, Group differences of the FA and MD metrics of the atlas-based tracts between hypertension and control groups. FDR, ${ }^{*} p<0.05$. ATR, Anterior thalamic radiation.

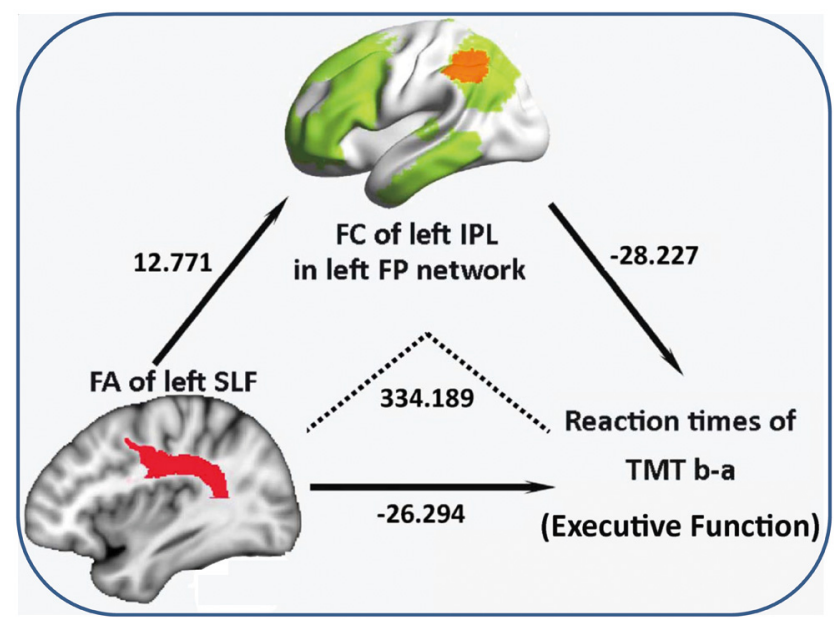

Figure 5. Mediation model illustrating the direct effect of FA left SLF on FC left inferior parietal lobe (IPL), the direct effect of FA left SLF on reaction times (RTs) of TMTba, the direct effect of FC left IPL on RTs of TMTba, and the mediating effect of FC on the association between FA left SLF and RTs of TMTba (i.e., effect of FA on TMT mediated through the effect of FA on FC left IPL). As indicated by the regression coefficients and the $p$ values, there was significant mediating effect of FC on the association between FA and TMT performance.

LFP network in hypertensive patients. Together, these results suggested that hypertension targets a specific pattern of cognitive declines and microstructural changes, which may help us understand why hypertension is a potential risk factor for dementia and cognitive decline.

There are many large population-based databases focused on the hypertension-related cognitive deficits over the world. These include the Framingham Heart Study (Ishihara et al., 2002), the Honolulu-Asia Aging Study (Bachoud-Lévi et al., 2000), the Kungsholmen Study (Hoffmann and Meyer, 2000), and the Rotterdam Scan Study (Rosenthal et al., 1999). These large-sample studies suggested that there is a decline in the ability to perform executive function (Hamanaka and Ohigashi, 1979; Lausberg et al., 1999) in hypertensive patients. There are a few large-sample studies about hypertension-related cognitive decline in patients of Han ethnicity (Lechevalier et al., 1977). However, the neuropsychological assessments only include general cognitive ability and memory tests.

Our results demonstrate that the hypertensive patients show decreased execution functions compared with the normotension patients, which is consistent with previous studies. We also found that there was a lower performance on one of the verbal memory tests in hypertensive patients. However, the photographic memory in hypertensive patients remained intact. The results also indicated that memory deficits were not very obvious in hypertensive patients. Hypertension was related to worse executive ability scores, which confirms the specific link between vascular disease and executive function and attention.

Based on the previous studies and our neuropsychological results, executive function and verbal memory are vulnerable in hypertension. Thus, we mainly focused on the LFP networks, the RFP networks, and the DMNs, which are related to these cognitive domains. There is a significant disruption in the LFP network between hypertension and controls under FDR correction. The number of studies on brain FC in patients with hypertension was very limited. Liang et al. have found that intrinsic functional network connectivity is significantly correlated with blood flow in healthy adults. Highly connected functional hubs require high metabolic support (Liang et al., 2013). Blood flow could be influenced by the changed vasculature in hypertension. Thus, the neuronal changes, vascular changes, or both types of changes are potentially of physiological significance to the functional network changes in hypertension.

Several lines of evidence suggest that the FP network investigated here plays a vital role in executive function, attention control, and working-memory processing (Leech et al., 2011), which is consistent with our neuropsychological results. First, it includes the frontal and parietal regions, which are activated by executive function-related tasks (Jennings et al., 1998; Niendam et al., 2012). Second, previous studies have reported that these regions are susceptible in hypertensive patients. Gray matter volume shrinkages (Gianaros et al., 2006) and reduced regional cerebral blood flow activation (Jennings et al., 1998; Gamalo et al., 2005) in these areas are common symptoms in hypertensive patients, even with successful control of blood pressure. Finally, the frontoparietal network overlaps considerably with brain regions targeted by stroke (Verma et al., 2004) and dementia (Tsioufis et al., 2006; Assmus et al., 2007), the quintessential disorder of executive function and attention.

Moreover, the differences were only found in the left hemisphere. The hemispheric differences may reflect subtle executive impairment. The left frontal activation was observed during the updating of the cognitive set, while right frontal activation was observed during the negative-feedback set (Delbeuck et al., 2003), which is more related with the reward system. However, previous studies rarely reported that hypertensive patients have reward sys- 
tem deficits. Thus, FP network asymmetry in hypertensive patients may represent differences in executive dysfunction.

Though there was also no significant difference in the DMNs between the two groups under FDR correction, under uncorrected conditions $(p<0.001)$ the right precuneus and left middle temporal gyrus showed significant differences. The DMN is important for episodic memory and social cognition (Leech et al., 2011), and is vulnerable to structural and functional deficits in dementia (Sugishita and Toyokura, 1978). Combining the evidence of cognitive deficits, the faint changes in DMN in hypertensive patients may suggest that hypertension is a harbinger of dementia. One previous study has demonstrated that head motion significantly affects measures of voxel-based or ROI-based FC (Van Dijk et al., 2012). Unlike voxel-based or ROI-based connectivity analyses, ICA is a data-driven approach, which is known for its ability to find noise-related components that represent head motion, physiological noise, eyeball movement, and other signal artifacts (Stevens et al., 2007).

Above all, hypertension mainly affects the LFP network and related cognitive domains. The alterations of FC in hypertensive patients seem to be very similar to those of vascular-related diseases (Corbetta et al., 2005; He et al., 2007).

In our study, decreased FA and increased MD of the left SLF, connecting the areas of FP networks, were found in hypertensive patients. Anatomically, the SLF overlaps with the parietal and the prefrontal components of the network (van den Heuvel et al., 2009a), which facilitates direct communication among these areas. SLF is especially important as it addresses higher-level cognitive functions and brain diseases. Our results suggest that the disrupted white matter integrity of the SLF could not enable information to travel quickly between the frontal and parietal regions.

Investigating white matter microstructural changes is vital to understanding the importance of hypertension in the occurrence of dementia and stroke (Salat et al., 2012). Reduced FA values in the SLF appeared in patients after stroke, which were correlated with a decreased ability to repeat spoken language (Lavados et al., 2002). Zarei et al. found that compared with AD, vascular dementia $(\mathrm{VaD})$ showed reduced FA, mainly in the SLF and forceps minor, illustrating how the nature and pattern of white matter tracts could be identified as a biomarker to differentiate $\mathrm{VaD}$ from AD (Nyffeler et al., 2003). Vascular injury may result in major white matter tract impairment (Godin et al., 2011; Maillard et al., 2012; Salat et al., 2012) and would reduce the efficacy of inter-regional neural communications (Kraut et al., 2008).

Though the right SLF was also impaired, there was no significant difference in RFP networks between hypertension and control patients. The impact of hypertension on the brain began from the left to right hemisphere. Generally, the left hemisphere of the brain is specialized for language abilities (Sugishita, 1979), and speech problems are very common in vascular disease (Orrison and Robertson, 1979); therefore, the left hemisphere is vulnerable in hypertension (Hrbek, 1979). Thus, when the white matter deficits were aggravated, the FC within the left hemisphere tended to change early.

Elaborating the relationships among white matter, FC, and cognitive function is very helpful to understand the neural mechanism of cognitive decline in hypertension. Based on the mediated analysis, we found that differences in FC in the LFP networks mediated SLF integrity reductions in executive function.

This neural model may describe why executive function impairment is very common in hypertension. As white matter disruption within SLF increased, the FP network was disrupted. The network could not integrate ongoing information from anatomically separate frontal and parietal regions very efficiently. Connectivity between frontal and parietal cortices appears to be critical for executive function (Rubens et al., 1977). Thus, executive function impairment is most common in hypertension.

Generally, previous studies have shown that reduced white matter integrity was significantly related with cognitive decline in healthy people and patients with vascular risks (O'Sullivan et al., 2005; Quinque et al., 2012; Caeyenberghs et al., 2014). The white matter changes may be very susceptible to hypertension. Early hypertension may result in the reduced integrity of white matter, even in midlife (Maillard et al., 2012). Gradually, hypertension also affects FC and cognitive ability. These results could explain how SLF integrity reductions influence the FC dysfunction and cognitive impairment in hypertensive patients.

Based on our results and a number of observations, there is a new view that reducing peripheral blood pressure typically fails to reverse the changes in brain function and structure (Jennings and Zanstra, 2009). Such patients are very susceptible to developing dementia. Thus, studies that evaluated the relationship between antihypertension treatment and dementia have yielded inconsistent results (Bautista, 2006; Shantha et al., 2009; Jiang et al., 2014).

\section{Limitations}

Our study has several limitations. First, before patients were diagnosed with hypertension, the white matter may have been affected by fluctuations in blood pressure (Maillard et al., 2012). However, this effect cannot be assessed accurately before diagnosis. Second, because the subjects were all from the community, their blood pressure could not be closely monitored in both mornings and evenings.

\section{Conclusions}

In summary, our results showed cognitive deficits, the associated white matter integrity reduction of SLF, and the FC alteration in the LFP network in the hypertension patients. Though additional studies are needed to further assess the neural mechanisms of hypertension-related cognitive decline even with orally reported blood pressure stably controlled, we support early intervention for hypertension and the need for additional means of neuroprotection.

\section{References}

Assmus A, Buss A, Milkereit EL, Meyer J, Fink GR (2007) Pure apraxic agraphia: a disconnection syndrome after left subcortical stroke. Eur J Neurol 14:e30-e31. Medline

Bachoud-Lévi AC, Ergis AM, Cesaro P, Degos JD (2000) Dissociation between distal and proximal left limb agraphia and agraphesthesia in a patient with a callosal disconnection syndrome. Cortex 36:351-363. CrossRef Medline

Bartolomeo P, Thiebaut de Schotten M, Doricchi F (2007) Left unilateral neglect as a disconnection syndrome. Cereb Cortex 17:2479-2490. CrossRef Medline

Bautista LE (2006) Associations of microalbuminuria with inflammation markers in hypertensive men. Am J Hypertens 19:467. CrossRef Medline

Buckner RL, Andrews-Hanna JR, Schacter DL (2008) The brain's default network: anatomy, function, and relevance to disease. Ann N Y Acad Sci 1124:1-38. CrossRef Medline

Bucur B, Madden DJ (2010) Effects of adult age and blood pressure on executive function and speed of processing. Exp Aging Res 36:153-168. CrossRef Medline

Caeyenberghs K, Leemans A, Leunissen I, Gooijers J, Michiels K, Sunaert S, Swinnen SP (2014) Altered structural networks and executive deficits in traumatic brain injury patients. Brain Struct Funct 219:193-209. CrossRef Medline 
Calhoun VD, Adali T, Pearlson GD, Pekar JJ (2001) A method for making group inferences from functional MRI data using independent component analysis. Hum Brain Mapp 14:140-151. CrossRef Medline

Corbetta M, Kincade MJ, Lewis C, Snyder AZ, Sapir A (2005) Neural basis and recovery of spatial attention deficits in spatial neglect. Nat Neurosci 8:1603-1610. CrossRef Medline

Davies NM, Kehoe PG, Ben-Shlomo Y, Martin RM (2011) Associations of anti-hypertensive treatments with Alzheimer's disease, vascular dementia, and other dementias. J Alzheimers Dis 26:699-708. CrossRef Medline

Delbeuck X, Van der Linden M, Collette F (2003) Alzheimer's disease as a disconnection syndrome? Neuropsychol Rev 13:79-92. Medline

Gamalo MA, Ombao H, Jennings JR (2005) Comparing extent of activation: a robust permutation approach. Neuroimage 24:715-722. CrossRef Medline

Gianaros PJ, Greer PJ, Ryan CM, Jennings JR (2006) Higher blood pressure predicts lower regional grey matter volume: consequences on short-term information processing. Neuroimage 31:754-765. CrossRef Medline

Godin O, Tzourio C, Maillard P, Mazoyer B, Dufouil C (2011) Antihypertensive treatment and change in blood pressure are associated with the progression of white matter lesion volumes: the Three-City (3C)-Dijon Magnetic Resonance Imaging Study. Circulation 123:266-273. CrossRef Medline

Gordon NG (1972) The Trail Making Test in neuropsychological diagnosis. J Clin Psychol 28:167-169. Medline

Hamanaka T, Ohigashi Y (1979) Disconnection syndrome: a critical analysis of its neuropsychological implications (author's transl) (in Japanese). No To Shinkei 31:907-912. Medline

Hayes AF (2013) Introduction to mediation, moderation, and conditional process analysis: a regression-based approach. New York: Guilford.

He BJ, Snyder AZ, Vincent JL, Epstein A, Shulman GL, Corbetta M (2007) Breakdown of functional connectivity in frontoparietal networks underlies behavioral deficits in spatial neglect. Neuron 53:905-918. CrossRef Medline

Hoffmann KT, Meyer BU (2000) Cystic dysplasia of the corpus callosum in interhemispheric disconnection syndrome. J Neurol Neurosurg Psychiatry 68:676-677. Medline

Hrbek V (1979) The Gerstmann syndrome as a component of a new broader syndrome of interparietal disconnection (in Czech). Cesk Neurol Neurochir 42:98-110. Medline

Ishiai S, Sugishita M, Ichikawa T, Gono S, Watabiki S (1993) Clock-drawing test and unilateral spatial neglect. Neurology 43:106-110. CrossRef Medline

Ishihara K, Nishino H, Maki T, Kawamura M, Murayama S (2002) Utilization behavior as a white matter disconnection syndrome. Cortex 38:379387. CrossRef Medline

Jennings JR, Zanstra Y (2009) Is the brain the essential in hypertension? Neuroimage 47:914-921. CrossRef Medline

Jennings JR, Muldoon MF, Ryan CM, Mintun MA, Meltzer CC, Townsend DW, Sutton-Tyrrell K, Shapiro AP, Manuck SB (1998) Cerebral blood flow in hypertensive patients: an initial report of reduced and compensatory blood flow responses during performance of two cognitive tasks. Hypertension 31:1216-1222. CrossRef Medline

Jiang S, Zhao R, Pan M, Venners SA, Zhong G, Hsu YH (2014) Associations of MTHFR and MTRR polymorphisms with serum lipid levels in Chinese hypertensive patients. Clin Appl Thromb Hemost 20:400-410. CrossRef Medline

Kelly AM, Uddin LQ, Biswal BB, Castellanos FX, Milham MP (2008) Competition between functional brain networks mediates behavioral variability. Neuroimage 39:527-537. CrossRef Medline

Knesevich JW, LaBarge E, Edwards D (1986) Predictive value of the Boston Naming Test in mild senile dementia of the Alzheimer type. Psychiatry Res 19:155-161. Medline

Koss E, Ober BA, Delis DC, Friedland RP (1984) The Stroop color-word test: indicator of dementia severity. Int J Neurosci 24:53-61. Medline

Kraut MA, Beason-Held LL, Elkins WD, Resnick SM (2008) The impact of magnetic resonance imaging-detected white matter hyperintensities on longitudinal changes in regional cerebral blood flow. J Cereb Blood Flow Metab 28:190-197. Medline

Kuo HK, Sorond F, Iloputaife I, Gagnon M, Milberg W, Lipsitz LA (2004) Effect of blood pressure on cognitive functions in elderly persons. J Gerontol A Biol Sci Med Sci 59:1191-1194. Medline

Lausberg H, Göttert R, Münssinger U, Boegner F, Marx P (1999) Callosal disconnection syndrome in a left-handed patient due to infarction of the total length of the corpus callosum. Neuropsychologia 37:253-265. CrossRef Medline

Lavados M, Carrasco X, Peña M, Zaidel E, Zaidel D, Aboitiz F (2002) A new sign of callosal disconnection syndrome: agonistic dyspraxia. A case study. Neurocase 8:480-483. CrossRef Medline

Le TH, Mukherjee P, Henry RG, Berman JI, Ware M, Manley GT (2005) Diffusion tensor imaging with three-dimensional fiber tractography of traumatic axonal shearing injury: an imaging correlate for the posterior callosal "disconnection" syndrome: case report. Neurosurgery 56:189. Medline

Lechevalier B, Andersson JC, Morin P (1977) Hemispheric disconnection syndrome with a "crossed avoiding" reaction in a case of MarchiafavaBignami disease. J Neurol Neurosurg Psychiatry 40:483-497. Medline

Leech R, Kamourieh S, Beckmann CF, Sharp DJ (2011) Fractionating the default mode network: distinct contributions of the ventral and dorsal posterior cingulate cortex to cognitive control. J Neurosci 31:3217-3224. CrossRef Medline

Liang X, Zou Q, He Y, Yang Y (2013) Coupling of functional connectivity and regional cerebral blood flow reveals a physiological basis for network hubs of the human brain. Proc Natl Acad Sci U S A 110:1929-1934. CrossRef Medline

Maillard P, Seshadri S, Beiser A, Himali JJ, Au R, Fletcher E, Carmichael O, Wolf PA, DeCarli C (2012) Effects of systolic blood pressure on whitematter integrity in young adults in the Framingham Heart Study: a crosssectional study. Lancet Neurol 11:1039-1047. CrossRef Medline

Mesulam MM (1998) From sensation to cognition. Brain 121:1013-1052. CrossRef Medline

Midorikawa A, Kawamura M, Takaya R (2006) A disconnection syndrome due to agenesis of the corpus callosum: disturbance of unilateral synchronization. Cortex 42:356-365. CrossRef Medline

Mok EH, Lam LC, Chiu HF (2004) Category verbal fluency test performance in Chinese elderly with Alzheimer's disease. Dement Geriatr Cogn Disord 18:120-124. Medline

Nielsen H (1975) Is constructional apraxia primarily an interhemispheric disconnection syndrome? Scand J Psychol 16:113-124. Medline

Niendam TA, Laird AR, Ray KL, Dean YM, Glahn DC, Carter CS (2012) Meta-analytic evidence for a superordinate cognitive control network subserving diverse executive functions. Cogn Affect Behav Neurosci 12: 241-268. CrossRef Medline

Nyffeler T, Bühler R, Höllinger P, Hess CW (2003) Reversible callosal disconnection syndrome in internal hydrocephalus. J Neurol Neurosurg Psychiatry 74:389-391. CrossRef Medline

Orrison WW, Robertson WC Jr (1979) Congenital ocular motor apraxia. A possible disconnection syndrome. Arch Neurol 36:29-31. Medline

O'Sullivan M, Barrick TR, Morris RG, Clark CA, Markus HS (2005) Damage within a network of white matter regions underlies executive dysfunction in CADASIL. Neurology 65:1584-1590. CrossRef Medline

Park KC, Jeong Y, Lee BH, Kim EJ, Kim GM, Heilman KM, Na DL (2005) Left hemispatial visual neglect associated with a combined right occipital and splenial lesion: another disconnection syndrome. Neurocase 11:310 318. CrossRef Medline

Quinque EM, Arélin K, Dukart J, Roggenhofer E, Streitbuerger DP, Villringer A, Frisch S, Mueller K, Schroeter ML (2012) Identifying the neural correlates of executive functions in early cerebral microangiopathy: a combined VBM and DTI study. J Cereb Blood Flow Metab 32:1869-1878. CrossRef Medline

Raz N, Rodrigue KM, Acker JD (2003) Hypertension and the brain: vulnerability of the prefrontal regions and executive functions. Behav Neurosci 117:1169-1180. Medline

Rosenberg SJ, Ryan JJ, Prifitera A (1984) Rey Auditory-Verbal Learning Test performance of patients with and without memory impairment. J Clin Psychol 40:785-787. Medline

Rosenthal G, Pomeranz S, Spektor S, Yacoub M, Israel ZH (1999) Syndrome of overdrainage associated with disconnection of a ventriculoperitoneal shunt. Pediatr Neurosurg 31:124-126. Medline

Rubens AB, Geschwind N, Mahowald MW, Mastri A (1977) Posttraumatic cerebral hemispheric disconnection syndrome. Arch Neurol 34:750-755. Medline

Salat DH, Williams VJ, Leritz EC, Schnyer DM, Rudolph JL, Lipsitz LA, McGlinchey RE, Milberg WP (2012) Inter-individual variation in blood 
pressure is associated with regional white matter integrity in generally healthy older adults. Neuroimage 59:181-192. CrossRef Medline

Schlösser R, Wagner G, Köhler S, Sauer H (2005) Schizophrenia as a disconnection syndrome. Studies with functional magnetic resonance imaging and structural equation modeling (in German). Radiologe 45:137-140, 142-143. CrossRef Medline

Sepe-Monti M, Pantano P, Vanacore N, De Carolis A, Bianchi V, Antonini G, Guidoni SV, Giubilei F (2007) Vascular risk factors and white matter hyperintensities in patients with amnestic mild cognitive impairment. Acta Neurol Scand 115:419-424. Medline

Shantha GP, Kumar AA, Bharadhi MK, Arthur P (2009) Role of gender in the associations of microalbuminuria with inflammatory markers in hypertensive subjects: a cross-sectional study. Kidney Blood Press Res 32: 434-439. CrossRef Medline

Sheridan LK, Fitzgerald HE, Adams KM, Nigg JT, Martel MM, Puttler LI, Wong MM, Zucker RA (2006) Normative Symbol Digit Modalities Test performance in a community-based sample. Arch Clin Neuropsychol 21: 23-28. Medline

Silva FA, Nina VJ, Santos AM, Figueredo Neto JA, Silva AA, Sousa FL, Figueredo ED, Salgado Filho N (2012) Echocardiographic associations with renal function in hypertensive individuals treated at primary care level (in Portuguese). J Bras Nefrol 34:161-169. CrossRef Medline

Sorg C, Riedl V, Mühlau M, Calhoun VD, Eichele T, Läer L, Drzezga A, Förstl H, Kurz A, Zimmer C, Wohlschläger AM (2007a) Selective changes of resting-state networks in individuals at risk for Alzheimer's disease. Proc Natl Acad Sci U S A 104:18760-18765. CrossRef Medline

Sperling RA, Dickerson BC, Pihlajamaki M, Vannini P, LaViolette PS, Vitolo OV, Hedden T, Becker JA, Rentz DM, Selkoe DJ, Johnson KA (2010) Functional alterations in memory networks in early Alzheimer's disease. Neuromolecular Med 12:27-43. CrossRef Medline

Stevens MC, Kiehl KA, Pearlson G, Calhoun VD (2007) Functional neural circuits for mental timekeeping. Hum Brain Mapp 28:394-408. CrossRef Medline

Sugishita M (1979) Disconnection syndrome (author's transl) (in Japanese). No To Shinkei 31:865-872. Medline

Sugishita M, Toyokura Y (1978) Disconnection syndrome (in Japanese). Nihon Rinsho [suppl]:1760-1761. Medline

Sun YW, Qin LD, Zhou Y, Xu Q, Qian LJ, Tao J, Xu JR (2011) Abnormal functional connectivity in patients with vascular cognitive impairment, no dementia: a resting-state functional magnetic resonance imaging study. Behav Brain Res 223:388-394. CrossRef Medline

Tsioufis C, Dimitriadis K, Taxiarchou E, Vasiliadou C, Chartzoulakis G, Tousoulis D, Manolis A, Stefanadis C, Kallikazaros I (2006) Diverse associations of microalbuminuria with C-reactive protein, interleukin-18 and soluble CD 40 ligand in male essential hypertensive subjects. Am J Hypertens 19:462-466. CrossRef Medline

Tupler LA, Welsh KA, Asare-Aboagye Y, Dawson DV (1995) Reliability of the Rey-Osterrieth Complex Figure in use with memory-impaired patients. J Clin Exp Neuropsychol 17:566-579. Medline

van den Heuvel MP, Mandl RC, Kahn RS, Hulshoff Pol HE (2009) Functionally linked resting-state networks reflect the underlying structural connectivity architecture of the human brain. Hum Brain Mapp 30:31273141. CrossRef Medline

Van Dijk KR, Sabuncu MR, Buckner RL (2012) The influence of head motion on intrinsic functional connectivity MRI. Neuroimage 59:431-438. CrossRef Medline

Verdelho A, Madureira S, Ferro JM, Basile AM, Chabriat H, Erkinjuntti T, Fazekas F, Hennerici M, O’Brien J, Pantoni L, Salvadori E, Scheltens P, Visser MC, Wahlund LO, Waldemar G, Wallin A, Inzitari D, Inzitari D (2007) Differential impact of cerebral white matter changes, diabetes, hypertension and stroke on cognitive performance among non-disabled elderly. The LADIS study. J Neurol Neurosurg Psychiatry 78:1325-1330. CrossRef Medline

Verma A, Singh NN, Misra S (2004) Transitory alexia without agraphia: a disconnection syndrome due to neurocysticercosis. Neurol India 52:378 379. Medline

Vincent FM, Sadowsky CH, Saunders RL, Reeves AG (1977) Alexia without agraphia, hemianopia, or color-naming defect: a disconnection syndrome. Neurology 27:689-691. CrossRef Medline

Weiler M, Teixeira CV, Nogueira MH, de Campos BM, Damasceno BP, Cendes F, Balthazar ML (2014) Differences and the relationship in default mode network intrinsic activity and functional connectivity in mild Alzheimer's disease and amnestic mild cognitive impairment. Brain Connect 4:567-574. CrossRef Medline

Wiseman RM, Saxby BK, Burton EJ, Barber R, Ford GA, O’Brien JT (2004) Hippocampal atrophy, whole brain volume, and white matter lesions in older hypertensive subjects. Neurology 63:1892-1897. CrossRef Medline 